\title{
Versatile setup for optical spectroscopy under high pressure and low temperature
}

\author{
Michaël K. Tran, ${ }^{1, a)}$ Julien Levallois, ${ }^{1}$ Ana Akrap, ${ }^{1}$ Jérémie Teyssier, ${ }^{1}$ Alexey B. Kuzmenko, ${ }^{1}$ \\ Florence Lévy-Bertrand, ${ }^{1,2}$ Riccardo Tediosi, ${ }^{1}$ Mehdi Brandt, ${ }^{1}$ Philippe Lerch, ${ }^{3}$ \\ and Dirk van der Marel ${ }^{1}$ \\ ${ }^{1}$ Département de Physique de la Matière Quantique, Université de Genève, Quai Ernest-Ansermet 24, \\ CH-1211 Genève 4, Switzerland \\ ${ }^{2}$ Institut Néel, 25 Avenue des Martyrs, BP166, 38042 Grenoble cedex 9, France \\ ${ }^{3}$ Infrared Beamline, Swiss Light Source, Paul Scherrer Institute, CH-5232 Villigen-PSI, Switzerland
}

(Received 23 February 2015; accepted 17 September 2015; published online 1 October 2015)

\begin{abstract}
We present an optical setup for spectroscopic measurements in the infrared and of Raman shift under high pressure and at low temperature. Using a membrane-driven diamond anvil cell, the pressure can be tuned in situ up to $20 \mathrm{GPa}$ and the temperatures ranges from room temperature down to $18 \mathrm{~K}$ in transmission mode and $13 \mathrm{~K}$ in reflection mode. In transmission, the setup is entirely working under vacuum to reduce the water absorption features and obtain a higher spectral stability. Since the infrared throughput obtained with a thermal source is limited, the use of a synchrotron source allowed to enhance the performance, as illustrated with results obtained with various materials. The analysis of the reflectivity is adapted so that it benefits from ambient pressure data and produces quantitative optical conductivity curves that can be easily compared to the results at ambient pressure. (C) 2015 AIP Publishing LLC. [http://dx.doi.org/10.1063/1.4931990]
\end{abstract}

\section{INTRODUCTION}

Temperature-dependent optical infrared and Raman spectroscopy allows one to probe low-energy vibrational, electronic, and magnetic excitations present in a large variety of systems. Applying pressure modifies the inter-atomic distances and it can also change the crystal structure of the material. Therefore, pressure can be thought of as a dial knob of the electronic band structure of materials. Thus, interactions between charge carriers can be probed, without the oftenunknown effects present when chemical doping is used.

However, such experiments are challenging, in particular, in view of the equipment needed to reach these extreme conditions: high pressure cells reaching several GPa limit the sample size way past sub-millimeter. In particular, this plays against the reachable spectral range in the far infrared due to diffraction limit and, most of all, limits the optical throughput thus rendering unavoidable the use of focusing optics and eventually the necessity to access a synchrotron light source. The optical throughput issue is in fact accentuated by the presence of diamond as both anvils and optical windows in the pressure cell (diamond anvil cell (DAC)). High pressure work at room temperature has become more accessible as an increasing number of infrared beamline are now equipped with DACs.

Another concern originates from the limited working distance (usually up to few centimeters) of commercially available infrared reflective objectives, which combined with the DAC's thickness, hinders the convenient placement of an optical windows fixed on a vacuum shroud that still allows the

a)Electronic mail: Michael.Tran@unige.ch precise positioning of DAC in the optical path. There are however groups getting round this problem, some using lower magnification coefficient objectives or custom-made reflective objectives with significant longer working distances (which also renders such objectives much bulkier and more delicate to integrate into a compact optical path) and other using DACs with a much lower profile (often at the expense of the in situ pressure tuning capability).

This space issue, combined with intrinsic difficulties of low temperature work at high pressure, renders high pressure optical studies difficult and reduced its diffusion as standard experimental method thus explaining the few reports of such work. Among them, the investigation of insulator-metal transitions in vanadium oxide compounds ${ }^{1}$ and hybridization in heavy-fermion $\mathrm{CeCoIn}_{5}{ }^{2}$ in the infrared, and solid ammonia ${ }^{3}$ and $\mathrm{MgB}_{2}{ }^{4}$ in Raman.

The lack of space around the DAC motivated us to conceive and build an optical setup in which both a membranedriven DAC and focusing optics are placed in a custom vacuum shroud designed for transmission measurements and allowing to reach low temperatures using a commercial cold-finger. Reflectivity measurements are done with a commercial Bruker infrared microscope in a low-profile custom-made shroud by a simple exchange of the cold-finger-DAC assembly.

Here, we describe in detail our optical, low-temperature high pressure experimental setup. Results obtained on liquid, powder, and single-crystal samples measured at the X01DC infrared beamline of the Swiss Light Source ${ }^{5}$ are briefly presented to illustrate the overall performance.

The unusual sample environment drastically complicates the data analysis and requires an elaborate calibration procedure which will be described further. 


\section{SETUP}

The description of the setup is divided in three parts. First, we describe the pressure cell, second, the cryogenic part, and finally, the coupling to the optical platform provided by the vacuum IR spectrometer or the nitrogen-purged IR microscope.

\section{A. Pressure application}

\section{General aspects}

In order to apply pressure to a sample under study, we use the well-established membrane DAC device. ${ }^{6}$ IR transparent diamonds (type IIa from Almax Industries) with $550 \mu \mathrm{m}$ diameter culets are squeezed together by a helium gas inflated membrane. We use a commercial model from BETSA as shown in Fig. 1. The sample under study is enclosed inside a thin ( $\gtrsim 10 \mu \mathrm{m}$ thick) gasket and placed in between the two diamond culets (see Figs. 1(b) and 2(b)). As the membrane inflates, the motion of the diamonds causes sealing of the chamber and produces the expected volume reduction from which a pressure increase is obtained.

\section{Cell preparation}

Gaskets are usually made out of small metallic discs. Several parameters are responsible for the actual sample thickness and the pressure range that a particular experiment can reach. The thickness of the disc, the material's hardness, the external pressure applied to the membrane, as well as the diameter of the hole drilled in the gasket, which forms the actual sample chamber, are main parameters. Gasketing is extensively discussed in the two articles of Dunstan and Spain. ${ }^{7,8}$

As an example, in our case, indented Copper Beryllium discs of $15 \mathrm{~mm}$ diameter and $150 \mu \mathrm{m}$ thickness were drilled by spark erosion with a tip of $200 \mu \mathrm{m}$ which produces sample chamber holes of about $250 \mu \mathrm{m}$ diameter.

\section{Pressure measurement}

It is impossible to predict the pressure within the cell solely by the knowledge of the pressure applied to the membrane. Thus, the pressure $P$ near the sample is determined by measuring the wavelength $\lambda$ of the $R_{1}$ ruby fluorescence's line, hence its pressure-temperature-dependent shift $\delta \lambda=\lambda$ $-\lambda_{\text {ref }}$ with respect to ambient conditions. We use an HR2000+ spectrometer from Ocean Optics optimized for fluorescence measurement with a $25 \mu \mathrm{m}$ slit and a $1800 \mathrm{~mm}^{-1}$ grating, a green $532 \mathrm{~nm} 15 \mathrm{~mW}$ laser as an excitation source, and a Lakeshore 332 for temperature control. The pressure in GPa is calculated ${ }^{9}$ with

$$
P\left(\delta \lambda, R_{1}\right)=1884\left(\frac{\delta \lambda_{p}}{R_{1}}\right)\left[1+5.5\left(\frac{\delta \lambda_{p}}{R_{1}}\right)\right],
$$

where $\delta \lambda_{p}=\delta \lambda-\delta \lambda_{T}$ is the only pressure-dependent contribution to the wavelength shift and $\delta \lambda_{T}$ is the temperature dependent shift expressed in $\AA$ as ${ }^{10}$

$$
\delta \lambda_{T}=\left\{\begin{array}{l}
-8.9, \quad \text { if } T<50 \mathrm{~K}, \\
\frac{6.67}{10^{2}} \Delta T+\frac{7}{10^{5}} \Delta T^{2}-\frac{2.3}{10^{7}} \Delta T^{3},
\end{array}\right.
$$

where $\Delta T=T-T_{\text {ref }}$ for $T \leq 300 \mathrm{~K}$ and $T_{r e f}$ is the temperature at which $\lambda_{\text {ref }}$ is taken at ambient pressure. The pressure can be determined in real-time thanks to an automated workflow (available upon request) performing in a loop the following operations: temperature and fluorescence measurements, multi-peaks fitting for the determination of the position of the $R_{1}$ line, and finally, the calculation of the pressure. ${ }^{11}$

\section{Sample environment}

Hydrostatic (or quasi-hydrostatic) conditions cannot be reached for samples in direct contact with both diamond anvils. Thus, samples are embedded by appropriate substances acting as pressure transmitting media. Liquids are the best choice for their ease of use and hydrostaticity. Because of their

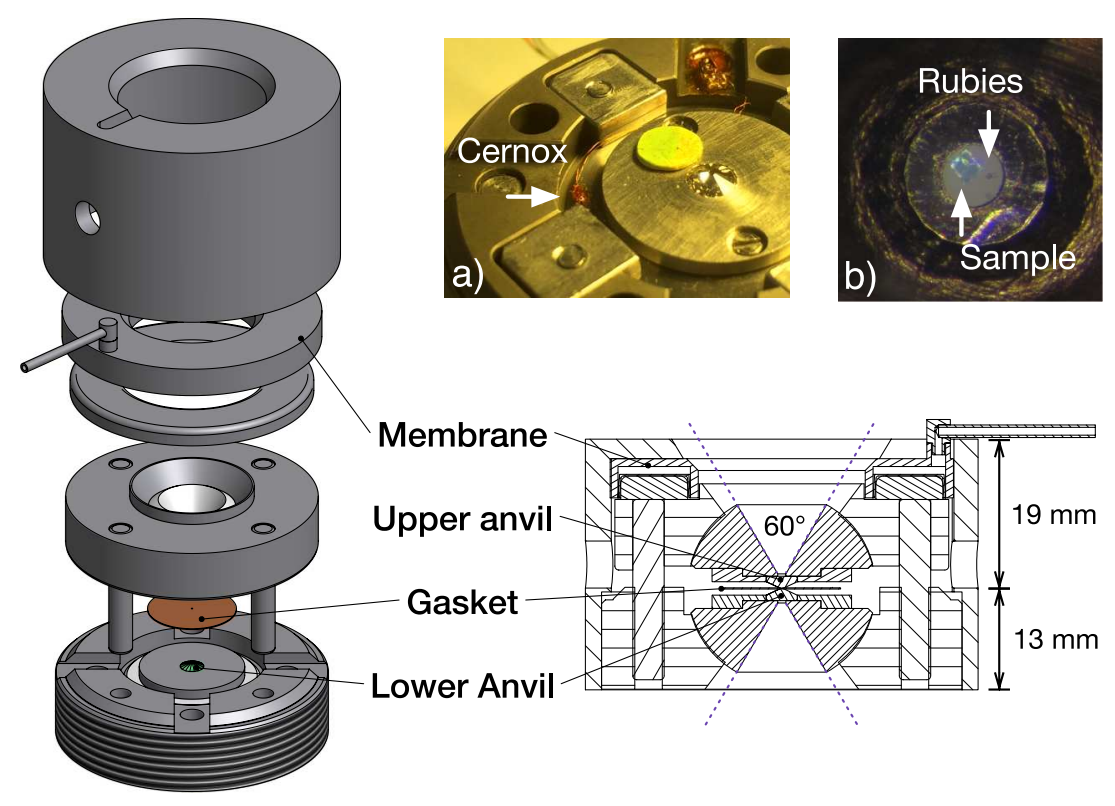

FIG. 1. BETSA DAC. (a) Cernox on the lower rocker. (b) Gasket's hole. 


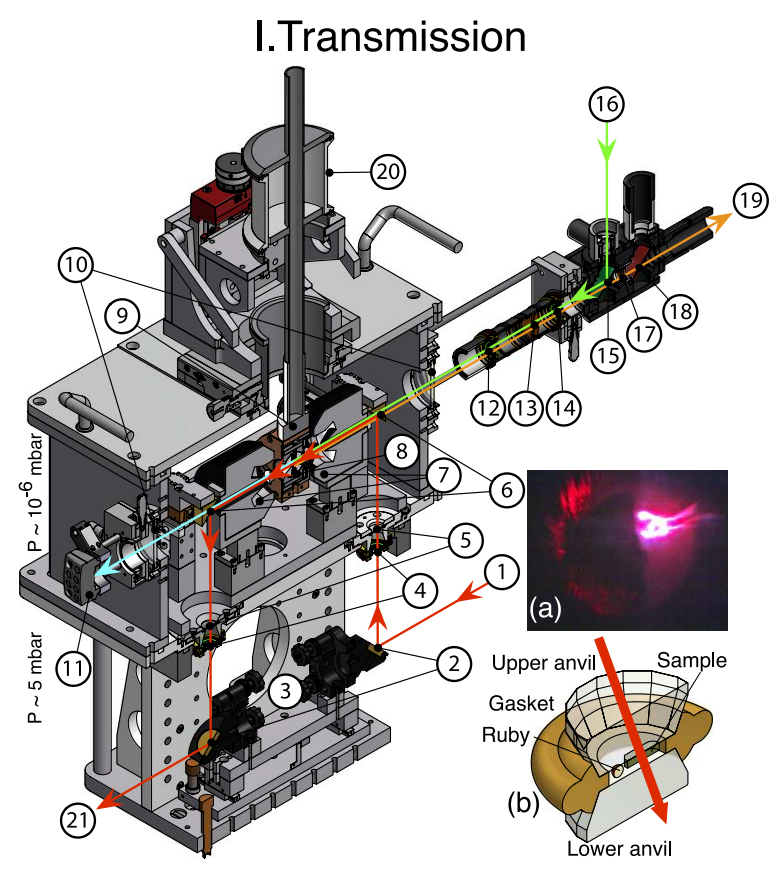

\section{II.Reflectivity}
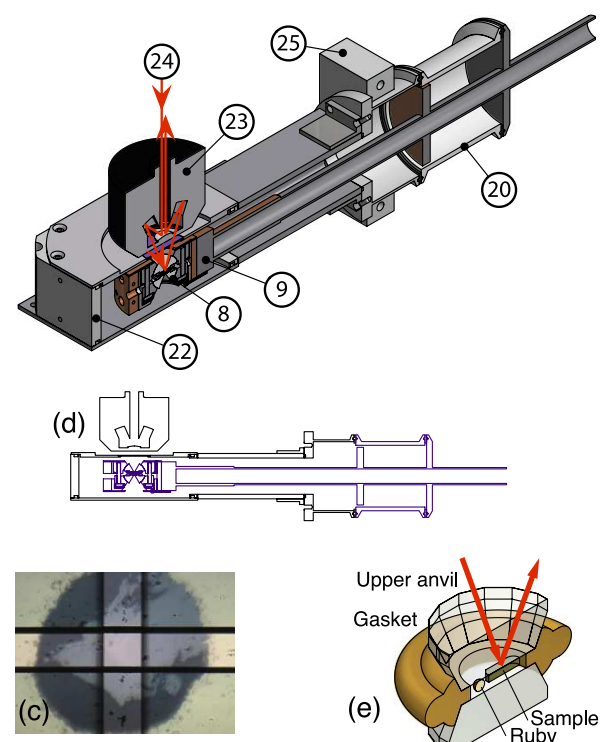

(e)

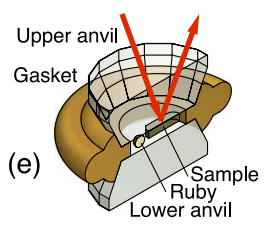

FIG. 2. Transmission setup and reflectivity setup in 3D cut view. Infrared is in red, laser excitation in green, ruby fluorescence in orange, and visible light collected by camera in blue. (I) Transmission. (a) Gasket hole with synchrotron light spot next to crystal sample (reference spectrum), as seen with camera (11). (b) Cut view of the sample environment with light passing through the sample (sample spectrum). (II) Reflectivity. (c) Hyperion's camera view of sample area delimited by field-stop blades (sample spectrum). (d) Shared parts (purple) with the transmission setup. (e) Sample environment in cut view with light reflected by the sample.

chemical composition infrared absorption can be strong in the MIR region, reducing their usefulness. However, if the spectral range of interest lies in the far infrared, Daphne oil 7474 is, for instance, believed to be a good candidate. ${ }^{12}$ The same is valid in the NIR above $4000 \mathrm{~cm}^{-1}$.

For our MIR experiments, we used $\mathrm{KBr}$ as a pressure medium. This mineral is transparent in the MIR region and is less prone to chemically react with the sample under study. Fine $\mathrm{KBr}$ powder was heated above $100{ }^{\circ} \mathrm{C}$ for at least $24 \mathrm{~h}$ to minimize water content.

One disadvantage of $\mathrm{KBr}$ (beside being driven away from hydrostatic conditions) is that loading a sample into the hole of the gasket is difficult: $\mathrm{KBr}$ grains agglomerate, tend to move the sample, and cannot always be distinguished easily from ruby chips and the entire loading process needs to be swift in order to limit water up-take. ${ }^{13}$ Moreover, the sealing of the gasket requires higher pressure applied to the membrane and the maximum pressure achieved with $\mathrm{KBr}$ is smaller than for loads using liquid pressure transmitting media. From the optical point of view, the presence of interfaces and gaps between grains of the medium causes a diffusive effect greatly decreasing the intensity of the signal in transmission geometry, as represented in Fig. 2(c), which lasts until the gains are more tightly put together with increasing pressure. This somewhat reduces the possibility of using transmission data as an input for a quantitative analysis under pressure. However, strong qualitative spectroscopic features can be easily followed during the pressure run. On the other hand, in reflectivity geometry where the sample kept in contact with one of the anvil as represented in Fig. 2(e) such diffusion is avoided as light does not pass through the pressure transmitting medium.

\section{B. Temperature measurement}

The DAC is clamped to the cold-finger of a commercially available liquid helium flow cryostat, model ST-100 from Janis. The stainless steel capillary line used to adjust the pressure into the membrane is thermally anchored with several windings along the thermal gradient of the cryostat. Temperature $T$ is monitored with two thermometers: one is placed on the coldfinger (original diode sensor) and another (small factor bare chip CX-1070-BC-4L Cernox sensor) inside the DAC attached on the lower rocker close to one of the diamonds as shown in Fig. 1(a). The lowest achieved temperature is $18 \mathrm{~K}$ and $13 \mathrm{~K}$ in transmission and reflection geometries, respectively.

\section{Optical paths}

\section{Transmission}

The transmission setup shown in Fig. 2(I) is designed to be placed directly into the sample compartment of a commercial Bruker FTIR $66 \mathrm{v} / \mathrm{S}$ spectrometer. The IR incoming (1) beam's nominal focal point (3) provided by this instrument is steered to the upper vacuum chamber of our setup and steered back to the nominal optical path of the spectrometer by two 1-inchdiameter gold plated mirrors on kinematic mounts (2). This pair of mirrors can be easily slid away using a translation stage in order to recover the original beam path, useful for initial adjustment. The guard vacuum of the cryogenic part of our setup needs to be better than the $\sim 5$ mbar reached by the spectrometer (due to the gas bearings of the interferometer scanner. Therefore, the upper chamber is separated from the compartment chamber by a pair of appropriate IR transparent windows (5): quartz, $\mathrm{KBr}$, and polypropylene for the NIR, 
MIR, and FIR spectral regions, respectively. At the equivalent focal point, there are variable size diaphragms (4) used to adjust the diameter of the illuminated area within the sample chamber inside DAC (8) in the focal plane of Cassegrain objectives (7). The beam is further steered into the right $15 \times$ magnification coefficient Cassegrain objective, numerical aperture 0.5 , mounted on a 3 -axis translation stage. The mirrors of this Cassegrain are aluminum-coated to also optimize the spectrum in the visible range for the fluorescence measurement. Original housing (20) of cold-finger (9) is attached to a custom-made $\mathrm{XYZ}$-manipulator, designed to withstand the force exerted by the atmospheric pressure, and used to position with precision the sample into the focal point delivered by the Cassegrain objective. It is of utmost importance to be able to move the DAC independently from the optics, in order to perform reference and sample measurements as shown in Figs. 2(a) and 2(b). On the left side of the DAC, the outgoing light is collected by second Cassegrain objective (7) with gold coated mirror and otherwise same specifications. The IR beam is then steered back into the original spectrometer's nominal beam path towards detector chamber (21). The combination of a heliumcooled bolometer and nitrogen-cooled MCT detectors allows to cover a spectral range from $50 \mathrm{~cm}^{-1}$ to $12000 \mathrm{~cm}^{-1}$ and the use of a silicon diode extends this range in the visible up to $25000 \mathrm{~cm}^{-1}$.

The Gold-coated $1 \mathrm{~cm}^{2}$ steering mirror (6) in front of each Cassegrain objective can be replaced (motorized linear translation stages) by a beamsplitter which is used to visualize the sample within the DAC with CCD camera (11) placed after quartz window (10). The translation stages can also completely clear the optical axis to perform the fluorescence measurement. This is done in the following way: the parallel laser beam exiting the collimator of optical fiber (16) is reflected by green dichroic mirror (15). Variable size diaphragm (14) allows tuning the intensity of the beam which is then collimated by $50 \mathrm{~mm}$ achromatic doublet (13) before passing through second size variable diaphragm (12) controlling the dimension of the laser spot entering into the right Cassegrain objective. The fluorescence light is collected in reflection and shares in reverse the same path up to the green dichroic mirror that is crossed by the red light which is then collimated by $50 \mathrm{~mm}$ achromatic doublet (17) before crossing red dichroic mirror (18) and finally being fed through another optical fiber (19) into to the HR2000+ spectrometer.

The optical components from (12) to (19) are mounted in a rigid ensemble whose position can be adjusted to match the rear focal point of the right reflective objective hence properly illuminating ruby inside the DAC.

\section{Reflectivity}

Although the high pressure infrared capabilities in reflectivity geometry have already been partially described in Ref. 14, where a Bruker Hyperion 3000 infrared microscope coupled to a Vertex 70V spectrometer is used in combination with a similar DAC, the reflectivity setup presented in Fig. 2(II) is actually an adaptation of DAC (8) clamped on coldfinger (9) housing ensemble (20) of Fig. 2(I) to the geometry of the Hyperion. Parts commonly shared with the transmission setup appear in purple in Fig. 2(d). Our home-made vacuum shroud (22) with a geometry of sufficiently low profile allows using the standard $15 \times$ reflective objective of microscope (23) with a working distance of about $24 \mathrm{~mm}$ thus keeping its original IR optical path (24). The positioning of the whole cryostat is achieved by a triple-axis transition stage allowing to precisely position the sample at the focal point of the condenser in order to make the sample and reference measurement. A coarse adjustment of the tilt along the axis of the cold finger is possible (25). On the Hyperion, the spectral range covered by a bolometer and MCT ranges from about $200 \mathrm{~cm}^{-1}$ to $8000 \mathrm{~cm}^{-1}$.

As described previously, ${ }^{14}$ ruby excitation is achieved by feeding a laser beam directly into the optical path of the infrared beam at the optical coupling of the spectrometer and the microscope. Fluorescence light is collected into an optical fiber through one of the ocular of the Hyperion and then analyzed by the Ocean Optics spectrometer.

This cryostat can also be used to perform Raman microspectroscopy under high pressure and at low temperature. An argon laser at a wavelength of $514.5 \mathrm{~nm}$ serves as a powertunable excitation source. The light passes through a beam expander and is then condensed at a confocal point of a Leica DM2500P microscope with an Olympus SLMPLN 20× objective mounted on its turret. The scattered light is collected in reflection mode and its Raleigh component is rejected with notch filters before being focused at the entrance slit of a half-meter monochromator. The grating (1800 grooves $/ \mathrm{mm}$ ) is mounted on a rotation stage allowing selection of the spectral range of interest. Detection of the dispersed light is performed by a 2048 pixels nitrogen-cooled CCD. Control over light polarization is achieved by two crossed linear polarizers that are placed before and after the microscope, respectively. A half-wave plate is placed after the first polarizer on a rotatable mount. $X X$ parallel polarization is obtained for an angle of $45^{\circ}$ between the axis of the first polarizer and the axis of the half-wave plate. $X Y$ crossed polarization is obtained when that angle is 0 . The ruby fluorescence is collected into an optical fiber through one of the binocular tube whereas the excitation light if brought by the other. The spectral range spans from about $50 \mathrm{~cm}^{-1}$ to $1400 \mathrm{~cm}^{-1}$ (with grating rotation).

\section{EXAMPLES OF MEASUREMENTS}

We present below some experiments conducted under high pressure using the described setup. The analysis performed on these examples is of increasing complexity because one needs to extract a more quantitative information on the pressure-induced changes on the optical conductivity.

\section{A. Liquid kerosene and $\mathrm{Sr}_{2} \mathrm{VO}_{4}$ powders}

Since kerosene is at the same time the sample and the medium of pressure, the entire surface of the gasket's hole can be illuminated by IR light ${ }^{15}$ and the calibration is thus done against an empty DAC spectrum. Although kerosene ${ }^{16}$ is not suitable for FIR measurements in transmission due to its strong absorption bands, ${ }^{17}$ it may be used in a higher energy range, for instance, above $5000 \mathrm{~cm}^{-1}$. The transmission spectrum shown 

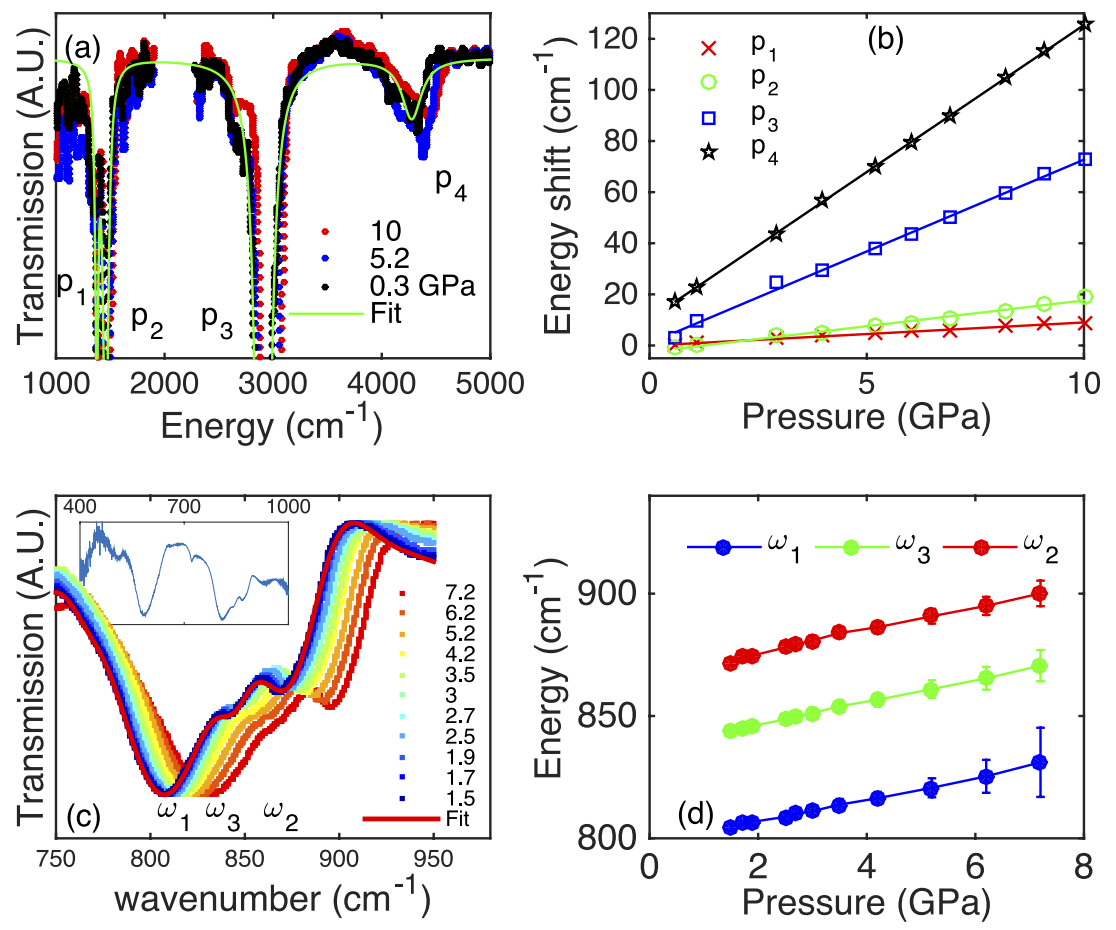

FIG. 3. (a) Pressure dependance of the MIR spectra of kerosene up to $10 \mathrm{GPa}$. (b) Shift of the position of four absorption peaks with pressure. (c) Transmission of $\mathrm{Sr}_{2} \mathrm{VO}_{4}$ up to $7 \mathrm{GPa}$. Inset displaying full $1.5 \mathrm{GPa}$ spectrum. (d) Pressure dependance of $\omega_{i=1,2,3}$.

in Fig. 3(a) can be fitted with Lorentzians to estimate their blueshift with pressure as displayed in Fig. 3(b).

Samples that cannot be grown in crystalline form are often produced in powders which are usually pressed into pellets prior to optical measurements at ambient pressure. In our case, we studied powders of tetragonal Mott insulator $\mathrm{Sr}_{2} \mathrm{VO}_{4}$. The blueshift of a series of absorption peaks under increasing pressure $P$ is shown in Fig. 3(c). Due to the very low transmission of this compound, it has been diluted by $\mathrm{KBr}^{18}$ The complex dielectric function $\epsilon(\omega, P)$ is modeled with a sum of DrudeLorentz (DL) oscillators,

$$
\epsilon(\omega, P)=\epsilon_{\infty}(P)+\sum_{i} \frac{\omega_{p, i}(P)^{2}}{\omega_{0, i}(P)^{2}-\omega^{2}-i \gamma_{i}(P) \omega},
$$

where the parameters $\epsilon_{\infty}(P), \omega_{p, i}(P)^{2}, \omega_{0, i}(P)^{2}$, and $\gamma_{i}(P)$ are, respectively, the high-frequency dielectric constant, the plasma frequency, the transverse frequency, and the linewidth of the $i$ th oscillator. Oscillators outside of the $760-900 \mathrm{~cm}^{-1}$ range are kept at their ambient-pressure values determined in a separate reflectivity experiment. ${ }^{19}$ The parameters are adjusted so that the measured transmission is well described by

$$
T(\omega, P)=\left|e^{i \omega \sqrt{\epsilon(\omega, P)} d / c}\right|^{2},
$$

where $c$ is the speed of light and $d$ the thickness of the $\mathrm{KBr}$ powders mixture.

The origin of two of these peaks $\left(\omega_{1}=808 \mathrm{~cm}^{-1}\right.$ and $\omega_{2}=874 \mathrm{~cm}^{-1}$ ) already seen in reflectivity ${ }^{19}$ has been the subject of theoretical investigation. ${ }^{20}$ It was suggested that a spin order parameter $\eta$ frozen at zero at low temperature and fluctuating between $[0, \pi]$ at room temperature influences the energy band levels. The two observed peaks were attributed to electronic transitions between these levels. However, the pressurized transmission spectrum shows also a third peak $\omega_{3}=836 \mathrm{~cm}^{-1}$, and all these peaks are shifting in the same way as shown in Fig. 3(d).

\section{B. Single crystals of BiTel}

High pressure measurements on sample in single crystal form are the closest one can get to a classic infrared spectroscopic measurement in condensed matter physics: one hopes to be able to treat the data in the same way. In the following, we illustrate this with a pressure study of BiTeI, which is a noncentrosymmetric semiconductor hosting up to now the largest Rashba spin splitting effect for a bulk material. ${ }^{21-23}$ Calculations suggest that the system might turn into a topological insulator by application of pressure via a progressive closing and re-opening of its energy gap. ${ }^{24}$ We conducted an optical high pressure study ${ }^{25}$ of this material that illustrates the usefulness of our setup. Transmission measurements at room temperature in Fig. 4(a) indicate a progressive reduction of the position of the maximum of transmission which lies just below the gap edge. Such behavior is also exhibited by the low temperature transmission in Fig. 4(b). As the low temperature data involved more than one sample, differences in amplitude arise and thus limit the interpretation to a qualitative level, but both indicate that pressure continuously reduces and eventually closes the energy gap. The reflectivity at room temperature is shown in Fig. 4(c). At low pressure, the reflectivity has a sharp plasma edge which redshifts with the application of pressure and disappears above $9.5 \mathrm{GPa}$ where the overall level of reflectivity increases. The low pressure curve shows oscillations in a transparent region above $0.4 \mathrm{eV}$ which are due to Fabry-Perot resonances inside the thin sample. As the transmission geometry is more prone to experimental artifacts 

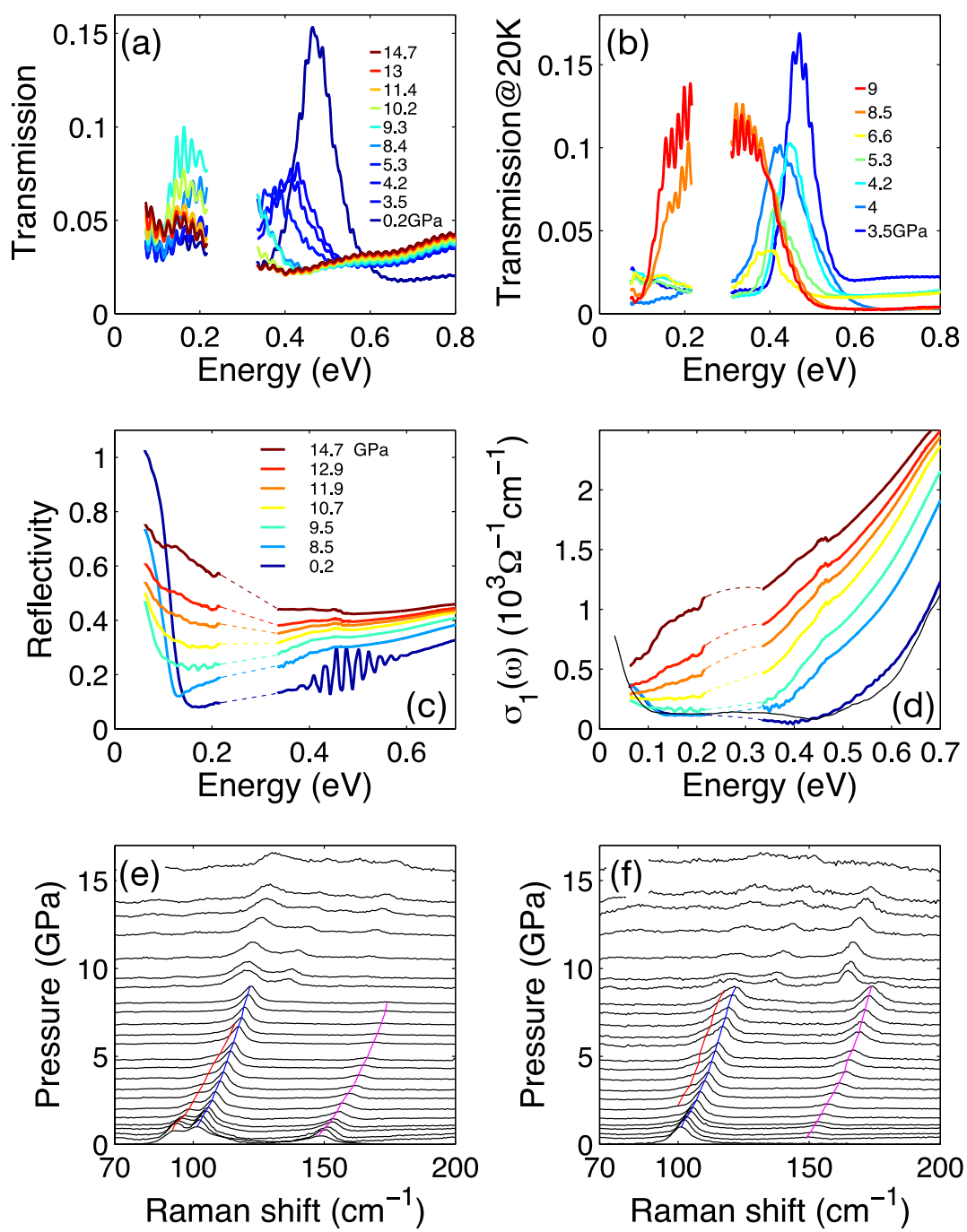

FIG. 4. BiTeI under High Pressure. Transmission spectrum at (a) room temperature and (b) at $20 \mathrm{~K}$. Region of the diamond absorption not shown. (c) Reflectivity $R_{s d}(\omega, P)$ and (d) real part of optical conductivity $\sigma_{1}(\omega, P)$. Dashed lines indicate the diamond absorption region. (e) Raman shift in $X X$ polarization under pressure and (f) $X Y$ polarization.

(mixture of light passing in and around the sample and thickness uncertainty, for instance), only the reflectivity data will be used as input for a quantitative analysis that we describe below.

\section{Data analysis}

As one measures the sample reflectivity within a DAC, one has to modify the method of analysis in order to account for the sample/diamond interface and also to compensate the renormalisation made against the spectrum of the gasket instead of a gold mirror. As was already pointed out by several studies, ${ }^{26-29}$ it is possible to perform a KramersKronig (KK) analysis based on the calculated reflectance at the sample/diamond interface

$$
R_{s d}(\omega, P)=\left|\frac{\sqrt{\epsilon_{d}(\omega)}-\sqrt{\epsilon(\omega, P)}}{\sqrt{\epsilon_{d}(\omega)}+\sqrt{\epsilon(\omega, P)}}\right|^{2},
$$

where $\epsilon_{d}(\omega)$ is the dielectric function of diamond, which for the limited spectral range discussed, is effectively constant and given by $\epsilon_{d}(\omega)^{1 / 2} \approx 2.4$. $\epsilon(\omega, P=0)$ is the dielectric function of the material obtained by KK transform at $P=0$ of the sample/vacuum absolute reflectivity $R_{0}(\omega)$ over the full energy range in order to numerically restore the phase of the reflectivity.

The presented method also starts with the calculation of $R_{s d}(\omega, P)$ in Equation (5) via the determination of $\epsilon(\omega, P)$ which is this time obtained using a KK consistent variational dielectric function ${ }^{30,31}$ (VDF) fit at $P=0$ reproducing all fine details of $R_{0}(\omega)$, equivalent to a standard KK transform. We assume that the dielectric function of the sample in the DAC at $P=0.2 \mathrm{GPa}$ (our lowest pressure curve) is very close to the dielectric function of the sample in vacuum $(P=0)$ which means that the reflectivity calculated and measured at the diamond interface should also be close. Therefore, we consider a scaling constant $C$ that is determined with the experimental reflectivity curves $R_{s d}^{e x p}(\omega, P=0.2)$ and $R_{s d}(\omega, P=0)$ which best satisfies our assumption

$$
R_{s d}^{e x p}(\omega, P=0.2)=C \cdot R_{s d}(\omega, P=0) .
$$

The introduction of $C$ also serves to compensate the absence of absolute normalisation. $C$ is assumed to be independent of 
pressure and therefore kept constant for all pressure curves. A variational fitting is carried out on $R_{s d}^{\exp }(\omega, P=0.2)$ by re-using the VDF solution for $R_{0}(\omega)$, while keeping fixed the oscillators outside of the measured spectral region of $R_{s d}^{\exp }(\omega, P=0.2)$. The recursive use of the VDF solution at the previous value of the pressure ensures the convergence of the procedure.

\section{Results}

The conductivity curve in Fig. 4(d) obtained with the lowest pressure is quantitatively close to the ambient one. The Fabry-Perot oscillations visible in the reflectivity curves (Fig. 4(c)) of the lowest pressures have been filtered out in the corresponding conductivity curves (Fig. 4(d)). As the pressure increases, one can follow the progressive filling of the gaped region up to a point where the overall level of conductivity increases. This combined with the transmission data unambiguously indicates a closing of the energy gap, but not its re-opening. Instead, this asymmetric behavior of the gap indicates that the high-pressure phase of the material is different. This structural transition is supported by the observation of discontinuities in the evolution of vibrational modes as seen in high-pressure polarized Raman spectroscopy, ${ }^{25}$ presented in Figs. 4(e) and 4(f). We conclude that the increase of the pressure above $9 \mathrm{GPa}$ does not produce a pressure-induced gap reopening, thus precluding the high-pressure topological state. An other high pressure study from Xi et al. ${ }^{32}$ also reported a change of structure seen in XRD data, but arrived at a different conclusion regarding the existence of a topological phase at lower pressure.

\section{SUMMARY AND CONCLUSION}

We presented an experimental setup allowing spectroscopic measurements under high pressure and at low temperature. It operates in both transmission and reflectivity geometry on separate spectrometers sharing the same coldfinger and DAC with in situ pressure tuning. Examples of highpressure optical data were presented and a quantitative method of infrared spectral analysis was described.

\section{ACKNOWLEDGMENTS}

We are grateful to Spiros Zanos, Daniel Chablaix, and Roland Pellet for technical assistance. Research was supported by the Swiss NSF through Grant Nos. 200020 and 135085, and NCCR MaNEP. We acknowledge the Swiss Light Source for the allocation of beam time under Proposal Nos. 20100801, 20110844, and 20120296 as well as for additional in-house beam time.
${ }^{1}$ A. Perucchi, L. Baldassarre, P. Postorino, and S. Lupi, J. Phys.: Condens. Matter 21, 323202 (2009).

${ }^{2}$ H. Okamura, A. Takigawa, E. D. Bauer, T. Moriwaki, and Y. Ikemoto, J. Phys.: Conf. Ser. 592, 012001 (2015).

${ }^{3}$ T. Kume, S. Sasaki, and H. Shimizu, J. Raman Spectrosc. 32, 383 (2001).

${ }^{4}$ A. F. Goncharov and V. V. Struzhkin, Phys. C: Supercond. 385, 117 (2003). ${ }^{5}$ PSI, X01DC beamline.

${ }^{6}$ R. Letoullec, J. P. Pinceaux, and P. Loubeyre, High Pressure Res. 1, 77 (1988).

${ }^{7}$ D. J. Dunstan and I. L. Spain, J. Phys. E: Sci. Instrum. 22, 913 (1989).

${ }^{8}$ I. L. Spain and D. J. Dunstan, J. Phys. E: Sci. Instrum. 22, 923 (1989).

${ }^{9}$ F. Datchi, A. Dewaele, P. Loubeyre, R. Letoullec, Y. Le Godec, and B. Canny, High Pressure Res. 27, 447 (2007).

${ }^{10}$ F. Occelli, Ph.D. thesis, Université Pierre et Marie Curie (Paris VI) and CEA, 2002.

${ }^{11}$ The fit process can be greatly accelerated by re-using the results of the previous fit as starting parameters when the fluorescence signal shows significantly shifted ruby lines.

${ }^{12}$ K. Murata, K. Yokogawa, H. Yoshino, S. Klotz, P. Munsch, A. Irizawa, M. Nishiyama, K. Iizuka, T. Nanba, T. Okada, Y. Shiraga, and S. Aoyama, Rev. Sci. Instrum. 79, 085101 (2008).

${ }^{13}$ Loading in a nitrogen purged glove box can partly solve this problem.

${ }^{14}$ P. Lerch, L. Quaroni, J. Wambach, J. Schneider, D. Armstrong, D. Rossetti, F. Mueller, P. Peier, V. Schlott, L. Carroll, P. Friedli, H. Sigg, S. Stutz, and M. Tran, J. Phys.: Conf. Ser. 359, 012003 (2012).

${ }^{15}$ For our pressure range the diameter of the hole is about $250 \mu \mathrm{m}$, a size large enough not to require synchrotron light.

${ }^{16}$ SIGMA-ALDRICH, Kerosene 329460 - reagent grade, low odor.

${ }^{17}$ H. Chung, M.-S. Ku, and J.-S. Lee, Vib. Spectrosc. 20, 155 (1999).

${ }^{18}$ Here the renormalization is made against the spectrum of a pressurized KBr-filled-DAC.

${ }^{19}$ J. Teyssier, R. Viennois, E. Giannini, R. M. Eremina, A. Günther, J. Deisenhofer, M. V. Eremin, and D. van der Marel, Phys. Rev. B 84, 205130 (2011).

${ }^{20}$ M. V. Eremin, J. Deisenhofer, R. M. Eremina, J. Teyssier, D. van der Marel, and A. Loidl, Phys. Rev. B 84, 212407 (2011).

${ }^{21}$ K. Ishizaka, M. S. Bahramy, H. Murakawa, M. Sakano, T. Shimojima, T. Sonobe, K. Koizumi, S. Shin, H. Miyahara, A. Kimura, K. Miyamoto, T. Okuda, H. Namatame, M. Taniguchi, R. Arita, N. Nagaosa, K. Kobayashi, Y. Murakami, R. Kumai, Y. Kaneko, Y. Onose, and Y. Tokura, Nat. Mater. 10, 521 (2011).

${ }^{22}$ A. Crepaldi, L. Moreschini, G. Autès, C. Tournier-Colletta, S. Moser, N. Virk, H. Berger, P. Bugnon, Y. J. Chang, K. Kern, A. Bostwick, E. Rotenberg, O. V. Yazyev, and M. Grioni, Phys. Rev. Lett. 109, 096803 (2012).

${ }^{23}$ G. Landolt, S. V. Eremeev, Y. M. Koroteev, B. Slomski, S. Muff, T. Neupert, M. Kobayashi, V. N. Strocov, T. Schmitt, Z. S. Aliev, M. B. Babanly, I. R. Amiraslanov, E. V. Chulkov, J. Osterwalder, and J. H. Dil, Phys. Rev. Lett. 109, 116403 (2012).

${ }^{24}$ M. S. Bahramy, B.-J. Yang, R. Arita, and N. Nagaosa, Nat. Commun. 3, 679 (2012).

${ }^{25}$ M. K. Tran, J. Levallois, P. Lerch, J. Teyssier, A. B. Kuzmenko, G. Autès, O. V. Yazyev, A. Ubaldini, E. Giannini, D. van der Marel, and A. Akrap, Phys. Rev. Lett. 112, 047402 (2014).

${ }^{26}$ S.-i. Kimura and H. Okamura, J. Phys. Soc. Jpn. 82, 021004 (2013); e-print arXiv: 1210.5310 [cond-mat.str-el].

${ }^{27}$ H. Okamura, J. Phys.: Conf. Ser. 359, 012013 (2012).

${ }^{28}$ A. Pashkin, M. Dressel, and C. A. Kuntscher, Phys. Rev. B 74, 165118 (2006).

${ }^{29}$ J. S. Plaskett and P. N. Schatz, J. Chem. Phys. 38, 612 (1963).

${ }^{30}$ A. B. Kuzmenko, Guide to Reffit: Software to Fit Optical Spectra (2004), available at http://optics.unige.ch/alexey/reffit_files/Manual.pdf.

${ }^{31}$ A. B. Kuzmenko, Rev. Sci. Instrum. 76, 083108 (2005).

${ }^{32}$ X. Xi, C. Ma, Z. Liu, Z. Chen, W. Ku, H. Berger, C. Martin, D. B. Tanner, and G. L. Carr, Phys. Rev. Lett. 111, 155701 (2013). 